
\title{
E Research Square \\ Label-Free Proteome Profiling for Lymphatic Metastasis in Papillary Thyroid Carcinoma
}

\section{Baolin Chen ( $\square$ baolin1995@foxmail.com )}

Affiliated Hospital of Zunyi Medical University https://orcid.org/0000-0002-6086-0841

Jiayang Li

Affiliated Hospital of Zunyi Medical University

\section{Yan Jiang}

Affiliated Hospital of Zunyi Medical University

Chengmin Luo

Affiliated Hospital of Zunyi Medical University

\section{Yuxiang Bao}

Affiliated Hospital of Zunyi Medical University

\section{Taolang Li}

Affiliated Hospital of Zunyi Medical University

\section{Xiaoming Cheng}

Affiliated Hospital of Zunyi Medical University

\section{Junyuan Lv}

Affiliated Hospital of Zunyi Medical University

\section{Research Article}

Keywords: Label-free quantitative proteomics, Papillary thyroid carcinoma, Differentially expressed protein, Biomarker· Bioinformatics

Posted Date: March 7th, 2022

DOI: https://doi.org/10.21203/rs.3.rs-1392853/v1

License: (9) This work is licensed under a Creative Commons Attribution 4.0 International License.

Read Full License 


\section{Abstract}

As the most common malignant tumor, the incidence of papillary thyroid carcinoma (PTC) has been significantly increased over the past few years. Approximately $30 \%-90 \%$ of patients with PTC present with cervical lymph node metastasis (LNM) at the time of initial diagnosis or follow-up, which has a serious impact on survival and prognosis. Six PTC N0 samples and six PTC N1b samples were collected. Proteins from the samples were analyzed by label-free quantitative proteomics. The bioinformatics of differentially expressed proteins (DEPs) was annotated with bioinformatic tools. The protein expression levels of the hub genes were verified using the HPA databases. Finally, we used the GEPIA database to perform survival analysis for the hub genes. A total of 1407 proteins were quantified under highly stringent criteria with fold change $\geq 2.0$ and $P<0.05$, and 95 DEPs were significantly changed between

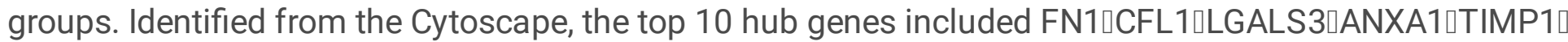
TPI1DMAPK1HSPA9ロACTR2 and S100A11. We determined the hub genes expression in thyroid normal and cancer tissues using the HPA database. The expression of FN1 and ANXA1 were closely related to disease free survival (DFS) and overall survival (OS) prognosis in PTC patients based on GEPIA database. These results revealed the pathways and mechanisms of LNM in PTC, thus providing a new perspective for the study of PTC with LNM. Together, these hub genes may have some clinical value in diagnosing PTC patients with LNM.

\section{Introduction}

The incidence rates of thyroid malignancies have dramatically risen over the last decade [1], with estimated 44,280 cases in the United States in 2021, making it the 7th most common new cancer diagnosed among women [2]. Papillary thyroid cancer (PTC) accounts for $75 \%-85 \%$ of all thyroid malignancies and usually has an excellent prognosis [3]. PTC mainly metastasizes through the cervical lymph node route, approximately $30 \%-90 \%$ of patients with PTC present with cervical lymph node metastasis (LNM) at initial diagnosis [4-7]. Research shows that LNM is an important risk factor for local recurrence and distant metastasis of PTC, which seriously affects the prognosis of patients [8]. Therefore, early diagnosis is vital for treatment and survival of the patients with PTC. Despite the strides made in the field of biomarkers for LNM in PTC, still it is far from perfection. If we can explore a molecular marker for accurate diagnosis PTC with cervical LNM, it will effectively help us develop treatment options.

Previous studies have analyzed PTC through microarrays [9], mRNA sequencing [10] as well as quantitative proteomics analysis [11]. Previous research had correlated certain genes, pathways and molecular processes to thyroid cancer [12]. Related genes expression in LNM of thyroid have been reported. However, the majority of the studies are based on transcriptome, there have been few proteomic studies in the field. Label-free quantitative proteomics technology has been used to study cancer pathogenesis, especially in the research on biomarkers related to cancer development [13]. The combination of quantitative proteomics and bioinformatics has proven to be a powerful tool to unravel the molecular mechanisms involved in the cancer process, which helps to deepen our understanding of 
the pathophysiology of cancer. The combination of proteomic and bioinformatic methods applied here have been described thoroughly elsewhere, including gastric [14], lung [15], breast [16]as well as cervical [17] cancers. These studies promote the application of proteomics in clinical research.

This study aims to improve the understanding of lateral LNM of PTC by screening key differential proteins and provide reference value for clinical diagnosis. Totally, 95 differentially expressed proteins (DEPs) was identified with the label-free quantitative proteomics technique by comparing protein expression profiles between PTC N0 samples as well as PTC N1b samples. DEPs were further analyzed in related pathways and mechanisms for LNM of PTC. Figure 1 showed the workflow of this study.

\section{Materials And Methods}

\section{Tissue sources}

A total of 12 PTC tissues obtained from Affiliated Hospital of Zunyi Medical University, including 6 cases with lateral LNM (N1b group) and 6 without metastasis (N0 group) (Table 1). No difference between the two groups with respect to the demographic variables of age, gender and race. This study was approved by the Medical Ethical Committee of the Affiliated Hospital of Zunyi Medical University, and all patients provided written informed consent.

\section{Label-free quantitative proteomics analysis}

\section{SDT lysis}

Firstly, SDT buffer was added into thyroid tissue. Subsequently, the lysate was sonicated, followed by boiling for $15 \mathrm{~min}$. After the centrifugation $(14,000 \mathrm{~g}, 40 \mathrm{~min})$, the supernatant was quantified by BCA Protein Assay Kit (Beyotime, CHINA) and stored at the temperature of $-80^{\circ} \mathrm{C}$.

\section{Sodium dodecyl sulfate polyacrylamide gel electrophoresis (SDS-PAGE) separation}

For each sample, proteins $(20 \mu \mathrm{g})$ were mixed with $6 \times$ loading buffer and boiled for 5 min. Subsequently, the proteins were separated on 12\% SDS-PAGE gel (constant voltage $250 \mathrm{~V}, 40 \mathrm{~min}$ ). The visualization of protein bands were achieved by Coomassie Blue R-250 staining.

\section{Filter-aided sample preparation (FASP digestion)}

For each sample, proteins $(200 \mu \mathrm{g})$ was add to dithiothreitol (DTT) until the final concentration was $100 \mathrm{mM}$ respectively and boiled for $5 \mathrm{~min}$, cooled to room temperature. Then $200 \mathrm{ul}$ UA buffer was added to mix and transfer to $30 \mathrm{KD}$ ultrafiltration centrifuge tube. After centrifuged at $14,000 \mathrm{~g}$ for $40 \mathrm{~min}$, discard filtrate and this process was repeated once. One hundred $\mu$ iodoacetamide (100 mM IAA in UA buffer) was added, followed by $600 \mathrm{rpm}$ oscillation for $1 \mathrm{~min}$. The incubation of the samples in darkness lasted for thirty minutes, followed by centrifugation $(12,500 \mathrm{~g}, 15 \mathrm{~min})$. Then, we added UA buffer (100 $\mu \mathrm{l})$, followed by the centrifugation $(12,500 \mathrm{~g}, 10 \mathrm{~min})$, and this process was repeated twice. A total of $100 \mu \mathrm{L}$ 
$40 \mathrm{mM} \mathrm{NH} 4 \mathrm{HCO} 3$ buffer was added into the mixture, followed by the centrifugation (12,500 $\mathrm{g}, 15 \mathrm{~min})$, and this process was repeated twice, the protein suspensions were digested with $40 \mu \mathrm{L}$ Trypsin buffer

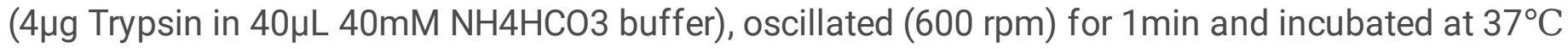
for 16-18h. Subsequently, a new collection tube was substituted, followed by centrifugation $(12,500 \mathrm{~g}, 15$ $\mathrm{min})$. Next, $20 \mu \mathrm{L} 40 \mathrm{mM} \mathrm{NH} 4 \mathrm{HCO} 3$ was added, followed by centrifugation $(12,500 \mathrm{~g}, 15 \mathrm{~min})$, and the filtrate was collected. The peptides of each sample were desalted on $\mathrm{C} 18$ Cartridges, and then redissolved in formic acid $(0.1 \%, 40 \mu \mathrm{l})$. The peptide content was quantified $\left(O D_{280}\right)$.

\section{Mass spectrometry}

Each sample was separated using an Easy- $n L C$ system (Thermo Fisher Scientific). The mobile phases were composed by $0.1 \%$ formic acid aqueous solution $(A)$ as well as $0.1 \%$ formic acid acetonitrile $(B)$. The chromatographic column was equilibrated with $100 \%$ liquid $A$, and the peptide mixture was loaded onto analytical column (Thermo Fisher Scientific, Acclaim PepMap RSLC 50um X 15cm, nano viper, $\mathrm{P} / \mathrm{N} 164943$ ) separated by an automatic sampler (flow rate: $300 \mathrm{nl} / \mathrm{min}$ ). The $2 \mathrm{~h}$ linear gradient elution was set as follows: 0-5 min, 3\% buffer B; 5-95 min, 3-28\% buffer B; $95-110$ min, 28-38\% buffer B; 110-115 min, $38-100 \%$ buffer $B ; 115-120$ min, $100 \%$ buffer $B$.

\section{LC-MS/MS analysis}

After separation by column chromatography, the samples were analyzed using a Q-Exactive Plus mass spectrometer for $120 \mathrm{~min}$. With the mass range of $350-1800 \mathrm{~m} / \mathrm{z}$ and the resolution of $70,000 \mathrm{at} \mathrm{m} / \mathrm{z}$ 200 , the MS spectra were acquired in the positive ion mode. The AGC target value was set at $3 e 6$ with maximum injection time of $50 \mathrm{~ms}$. The mass-charge ratios of polypeptide and polypeptide fragments were collected as followings: 10 fragment mapswere was collected after each full scan (MS2 scan, HCD); Isolation window was $2 \mathrm{~m} / \mathrm{z}$, secondary resolution was 17,500 , the normalized collision energy was $27 \mathrm{eV}$ and the maximum injection time was $45 \mathrm{~ms}$.

\section{Data analysis}

Database search and LFQ were performed with the software MaxQuant (version 1.5.5.1). Relevant parameters and descriptions are as follows: Enzyme $=$ Trypsin, Main search $=4.5 \mathrm{ppm}, \mathrm{MS} / \mathrm{MS}$ Tolerance $=20$ ppm,Max Missed Cleavages = 2, First search $=20$ ppm, Protein FDR $\leq 0.01$, Peptide FDR $\leq 0.01$.

\section{Bioinformatic analysis}

The differentially expressed proteins (DEPs) in N0 group compared to N1b were identified with Pख0.05 was considered significant. In order to study the changes in DEPs associated with lateral LNM in thyroid cancer, it is necessary to understand the molecular function (MF), cellular components (CC) as well as biological processes (BP) of each protein. Matplotlib software was applied to classify the expressions of samples and proteins simultaneously so as to examine the rationality of the selected target proteins. The relationship among different genes was explored through gene Ontology (GO) classification. The KOALA 
(KEGG Orthology And Links Annotation) software was used for KEGG pathway predictions. Knowledge on the subcellular localization of a protein is important for the inference of its biological effect. Therefore, we used the software WoLF PSORT to predict the subcellular localization of the DEPs.

STRING(https://string-db.org/) was use for the protein-protein interaction (PPI) network analysis of DEPs to understand the relationship between genes. The modules in the PPI network were analyzed by the cytoHubba plug in Cytoscape software, and the top 10 hub genes were determined. Complying with the degree algorithm, the top 10 DEPs highly connected in the gene co-expression network were selected as hub gene.

\section{Human Protein Atlas (HPA) database}

HPA(http://www.proteinatlas.org/) is consisted by the information of 26,000 human proteins on the tissues as well as cells. The protein level of these hub genes among tissues of thyroid cancer as well as normal thyroid were analyzed in HPA online database.

\section{Prognosis analysis}

The association between patient prognosis and hub genes were analyzed using the GEPIA, which is an Online Biomarker Validation Tool and Database for Cancer Gene Expression Data Using Survival Analysis.

\section{Results}

\section{Identification of DEPs between NO group and N1b group}

We perfomed a comprehensive analysis of the overall protein of tissue specimens. Proteomic profiling was conducted using a label-free LC-MS/MS. Based on proteomics, 1407 proteins were identified, among which 95 proteins were significantly different in the expression levels between N0 group and N1b group, including 68 up-regulated proteins and 27 down-regulated proteins. The volcano map was drawn by the differential expression rate and $P$ value, showing the proteins with statistical difference between the two groups (Fig.2).

\section{Hierarchical clustering analysis (HCA)}

To assess the rationality of grouping and evaluate whether the N0 group could be distinguished from N1b group by the variation of DEPs, HCA was used to analyze the expression patterns of DEPs in groups. HCA was performed for DEPs (Fig.3). The expression patterns of DEPs in samples was similar within the same group but significantly different between groups, suggesting that the DEPs were able to significantly distinguish the N0 group from N1b group.

\section{Protein Functional Enrichment Analysis}


To study the changes of DEPs between N0 and N1b group, it is necessary to understand the MF, CC as well as BP in cells. As a bioinformatics platform, Blast2Go was used to perform $\mathrm{GO}$ function annotation as well as enrichment analysis. The enriched $\mathrm{GO}$ terms, including $\mathrm{BP}, \mathrm{CC}$, as well as MF, were shown in Fig.4. The BP classification of GO analysis demonstrated that the DEPs obviously enriched the regulation of plasminogen activation, growth plate cartilage development and neutrophil degranulation. For CC, these DEPs are rich in collagen trimer, postsynaptic density and ion channel complex. In addition, they are significantly rich in calcium channel activity, phospholipase A2 inhibitor activity and ion channel binding in the MF category.

\section{Pathway analysis}

DEPs are involved 216 pathways, and 26 pathways showed significant difference. Fig. 5 showed the top ten KEGG terms which were the most significantly enriched. Specifically, the majority of pathways were PI3K-Akt signaling pathway, FcyR-mediated phagocytosis, and TGF- $\beta$ signaling pathway.

\section{Subcellular localization prediction}

In this study, the WoLF PSORT software were used to predict the subcellular localization of the 95 DEPs. In total, $48.4 \%(n=46)$ were localized in the cytosol, $18.9 \%(n=18)$ in the nucleus, $16.8 \%(n=16)$ in the mitochondria,9.5\%(n=9) in the extracellular, $5.3 \%(n=5)$ in the plasma membrane and $1.1 \%(n=1)$ in the endoplasmic (Fig.6). The results suggest that DEPs were mainly located in the cytoplasm.

\section{Integration of PPI network and module analysis}

Organisms depend on proteins to achieve their functions, among which PPI exerts acritical effect on the pathogenesis of diseases. Based on the STRING online database, the 95 integrated DEPs were analyzed; besides, the PPI network was established (Fig.7). Next, we used the cytoHubba in Cytoscapeto find the top 10 hub genes. and the identified hub genes were FN1, CFL1, LGALS3, ANXA1, TIMP1, TPI1, MAPK1, HSPA9, ACTR2, S100A11 (Fig.8).

\section{Validation of hub genes among PTC patients}

In order to verify the protein expressions of hub genes among thyroid tissues, we employed HPA database to obtain immunohistochemical staining (Fig.9). Then, protein expression levels of the hub genes were found to be remarkably up-regulated in tumor tissues than normal tissues.

\section{Prognostic significance of hub genes in PTC patients}

To explore the prognostic effects of the top 10 hub genes on thyroid cancer, survival analysis was performed using the survival information of the GEPIA database. Next, we explored the association between gene expression and survival, and found the FN1 was correlated with the disease-free survival (DFS) of thyroid cancer. Compared to that with low FN1 expression, DFS was found to be lower in the group with high FN1 expression. In addition, the ANXA1 was also found to be correlated with Overall 
Survival (OS) of thyroid cancer. OS was higher in the group with high ANXA1 expression than that with the low ANXA1 expression (Fig.10).

\section{Discussion}

Despite the majority of patients with PTC demonstrate excellent clinical outcomes, $20 \%-30 \%$ of PTC patients still develop regional recurrence or distant metastasis, which is mainly related to LNM at the initial diagnosis [18]. Therefore, new prognostic biomarkers are in need for PTC patients. There have been a few reports on the key genes of LNM in PTC. Zhang et al. identified differentially expressed genes and signaling pathways involved in LNM of PTC [19]. However, protein is the direct executor of gene function, and protein is much more stable than RNA, which is easy for clinical detection. There are few studies on the mechanisms of LNM from protein level. The quantitative proteomics combined with bioinformatics may help to reveal signaling pathways related to LNM in PTC. By studying the DEPs of PTC with or without LNM, more pathological and biological information can be obtained, and then an effective biomarker can be explored.

In our study, label-free quantitative proteomics technology was used to analyze the tissue of PTC with or without lateral LNM. The further screening revealed 95 DEPs between the two groups. The investigation of the subcellular localization of DEPs were predicted by WoLF PSORT software. The results showed that the DEPs were mainly located in the cytoplasm, which further suggested that they were the key effect factors for regulating LNM. Next, we performed a series of bioinformatics analysis and identified the following 10 hub genes: FN1, CFL1, LGALS3, ANXA1, TIMP1, TPI1, MAPK1, HSPA9, ACTR2 and S100A11. The HPA database was searched to confirm the high expression of these genes in PTC tissues.

To further analyze how hub genes linked with survival of PTC patients, a prognostic analysis was carried out for the hub genes based on GEPIA online database. The results showed that the expressions of FN1 as well as ANXA1 were related to DFS and OS respectively. FN1(Fibronectin 1) encodes the extracellular matrix protein fibronectin, which plays crucial roles in cell adhesion, migration processes, growth, and metastasis. Many studies have reported that regulation of FN1 in malignant tumors, including gastric [20], cervical [21], as well as colorectal cancers [22]. However, the FN1 gene was found to be a key regulator in PTC development. Tripartite motif protein 29(TRIM29) promote the invasion and metastasis of PTC cells by up-regulating the expression of FN1 [23]. Furthermore, studies indicate that TGM2 activated FN1 through NF-kb to promote the invasion as well as metastasis of PTC [24]. Therefore, these findings highlight the FN1 gene as potential target for anti-metastasis therapy, which provides certain value for clinical treatment. Sponziello et al. confirmed that the proliferation, adhesion, migration, as well as invasion of PTC cells were significantly suppressed after the silencing of FN1 [25]. Ursolic acid inhibited PTC cell viability, proliferation, and EMT but promoted apoptosis via suppressing FN1, thus exert antitumor effects in vivo [26]. As a calcium-dependent phospholipid-binding protein, ANXA1(Annexin A1) presents a molecular weight of $37 \mathrm{kDa}$ and is involved in cell growth, differentiation, and apoptosis [27, 28]. As reported in previous studies, ANXA1 expression was related with differentiation of thyroid carcinoma cells. High expression of ANXA1 in differentiated thyroid carcinoma while undifferentiated 
thyroid carcinoma cells had low expression of ANXA1[29]. CCK8 proliferation and flow cytometry apoptosis experiment confirmed that in PTC patients, MYC (proto-oncogene) could regulated the high expression of ANXA1 and promote the proliferation of PTC cells [30]. Furthermore, as a key oncogene during PTC carcinogenesis, ANXA1 could enhance the proliferation and migration of tumors by regulating EMT signaling pathway as well as IL-6/JAK2/STAT3 in PTC [31]. Further study the relationship between ANXA1 and prognosis of PTC. Studies used a Cox-PH regression analysis to find ANXA1 that was closely related to overall survival (OS) of patients with PTC [32]. On the other hand, studies on ANXA1 in other cancers have also been reported. ANXA1 promotes nasopharyngeal carcinoma growth and metastasis via binding and stabilization of EphA2[33]. At the same time, ANXA1 interacts with formyl peptide receptor and promotes MMP2 expression through MAPK and STAT3 pathways, thereby promoting melanoma cell metastasis [34]. Our study confirmed the association of ANXA1 expression level with OS in patients with PTC, similar to previous studies. It is suggested that ANXA1 is a key gene in LNM of PTC.

\section{Conclusion}

In this work, we combined proteomics and bioinformatics analysis to explore the key genes of PTC lateral LNM and prognosis. In conclusion, FN1 and ANXA1 may have the potential as candidate biomarkers for lateral LNM and tumor recurrence among PTC.

\section{Declarations}

Author contributions All authors contributed in this project.

Funding This study was funded by National Natural Science Foundation of China (No.81860715) and Doctor Foundation of Affiliated Hospital of Zunyi Medical University (No.201712).

Data availability The original contributions presented in the study are included in the article. Further inquiries can be directed to the corresponding author.

Conflict of interest The authors declare that they have no competing interests.

Ethics statement The studies involving human participants were reviewed and approved by the ethics committee of the Affiliated Hospital of Zunyi Medical University, and informed consent of all participants was obtained.

\section{References}

1 Vaccarella S, Dal Maso L, Laversanne M, Bray F, Plummer M, Franceschi S. The Impact of Diagnostic Changes on the Rise in Thyroid Cancer Incidence: A Population-Based Study in Selected High-Resource Countries. Thyroid. 2015;25(10):1127-36.

2 Siegel RL, Miller KD, Fuchs HE, Jemal A. Cancer Statistics, 2021. CA Cancer J Clin. 2021;71(1):7-33. 
3 Lim H, Devesa SS, Sosa JA, Check D, Kitahara CM. Trends in Thyroid Cancer Incidence and Mortality in the United States, 1974-2013. JAMA. 2017;317(13):1338-1348.

4 Liu C, Xiao C, Chen J, Li X, Feng Z, Gao Q, et al. Risk factor analysis for predicting cervical lymph node metastasis in papillary thyroid carcinoma: a study of 966 patients. BMC Cancer. 2019;19(1):622.

5 He M, Lin C, Yin L, Lin Y, Zhang S, Ma M. Value of Dual-Energy Computed Tomography for Diagnosing Cervical Lymph Node Metastasis in Patients With Papillary Thyroid Cancer. J Comput Assist Tomogr. 2019;43(6):970-975.

6 Wang J, Jiang X, Xiao G, Zhou W, Hu Y. Excellent diagnostic performance of FNA-Tg in detecting lymph nodes metastases from papillary thyroid cancer. Future Oncol. 2020;16(33):2735-2746.

7 Lee YM, Sung TY, Kim WB, Chung KW, Yoon JH, Hong SJ. Risk factors for recurrence in patients with papillary thyroid carcinoma undergoing modified radical neck dissection. Br J Surg. 2016;103(8):1020-5.

8 Feng JW, Qin AC, Ye J, Pan H, Jiang Y, Qu Z. Predictive Factors for Lateral Lymph Node Metastasis and Skip Metastasis in Papillary Thyroid Carcinoma. Endocr Pathol. 2020;31(1):67-76.

9 Wang Q, Shen Y, Ye B, Hu H, Fan C, Wang T, et al. Gene expression differences between thyroid carcinoma, thyroid adenoma and normal thyroid tissue. Oncol Rep. 2018;40(6):3359-69.

10 Haase J, Misiak D, Bauer M, Pazaitis N, Braun J, Pötschke R,et al. IGF2BP1 is the first positive marker for anaplastic thyroid carcinoma diagnosis. Mod Pathol. 2021; 34(1):32-41.

11 Wei X, Zhang Y, Yu S, Li S, Jiang W, Zhu Y, et al. PDLIM5 identified by label-free quantitative proteomics as a potential novel biomarker of papillary thyroid carcinoma. Biochem Biophys Res Commun. 2018;499(2): 338-44.

12 Bi W, Huang J, Nie C, Liu B, He G, Han J,et al. CircRNA circRNA_102171 promotes papillary thyroid cancer progression through modulating CTNNBIP1-dependent activation of $\beta$-catenin pathway. $J$ Exp Clin Cancer Res. 2018;37(1):275.

13 Kumar V, Ray S, Ghantasala S, Srivastava S. An Integrated Quantitative Proteomics Workflow for Cancer Biomarker Discovery and Validation in Plasma. Front Oncol. 2020;10:543997.

14 Liu W, Yuan J, Liu Z, Zhang J, Chang J. Label-Free Quantitative Proteomics Combined with Biological Validation Reveals Activation of Wnt/ $\beta$-Catenin Pathway Contributing to Trastuzumab Resistance in Gastric Cancer. Int J Mol Sci. 2018;19(7):1981.

15 Lu M, Chen W, Zhuang W, Zhan X. Label-free quantitative identification of abnormally ubiquitinated proteins as useful biomarkers for human lung squamous cell carcinomas. EPMA J. 2020; 11(1):73-94. 
16 Gomig THB, Gontarski AM, Cavalli IJ, Souza RLR, Lucena ACR, Batista M, et al. Integrated analysis of label-free quantitative proteomics and bioinformatics reveal insights into signaling pathways in male breast cancer. Genet Mol Biol. 2021; 44(1):e20190410.

17 Zhang L, Wang W, Zhang S, Wang Y, Guo W, Liu Y,et al. Identification of lysine acetylome in cervical cancer by label-free quantitative proteomics. Cancer Cell Int. 2020;20:182.

18 Brown RL, de Souza JA, Cohen EE. Thyroid cancer: burden of illness and management of disease. J Cancer. 2011;2:193-9.

19 Zhang Z, Zhao S, Wang K, Shang M, Chen Z, Yang H, et al. Identification of biomarkers associated with cervical lymph node metastasis in papillary thyroid carcinoma: Evidence from an integrated bioinformatic analysis. Clin Hemorheol Microcirc. 2021;78(2):117-126.

20 Wu C, Zeng MH, Liao G, Qian K, Li H. Neuropilin-1 Interacts with Fibronectin-1 to Promote EpithelialMesenchymal Transition Progress in Gastric Cancer. Onco Targets Ther. 2020;13:10677-87.

21 Zhou Y, Shu C, Huang Y. Fibronectin promotes cervical cancer tumorigenesis through activating FAK signaling pathway. J Cell Biochem. 2019;[Epub]: doi: 10.1002/jcb.28282.

22 Ye Y, Zhang R, Feng $\mathrm{H}$. Fibronectin promotes tumor cells growth and drugs resistance through a CDC42-YAP-dependent signaling pathway in colorectal cancer. Cell Biol Int. 2020;44(9):1840-1849.

23 Wu T, Zhang DL, Wang JM, Jiang JY, Du X, Zeng XY, et al. TRIM29 inhibits miR-873-5P biogenesis via CYTOR to upregulate fibronectin 1 and promotes invasion of papillary thyroid cancer cells. Cell Death Dis. 2020; $11(9): 813$.

24 Sun W, Qin Y, Wang Z, Dong W, He L, Zhang T, et al. The NEAT1_2/miR-491 Axis Modulates Papillary Thyroid Cancer Invasion and Metastasis Through TGM2/NFkb/FN1 Signaling. Front Oncol. 2021;11: 610547.

25 Sponziello M, Rosignolo F, Celano M, Maggisano V, Pecce V, De Rose RF, et al. Fibronectin-1 expression is increased in aggressive thyroid cancer and favors the migration and invasion of cancer cells. Mol Cell Endocrinol. 2016;431:123-32.

26 Cao M, Xiao D, Ding X. The anti-tumor effect of ursolic acid on papillary thyroid carcinoma via suppressing Fibronectin-1. Biosci Biotechnol Biochem. 2020;84(12):2415-2424.

27 Correia CT, Conceição IC, Oliveira B, Coelho J, Sousa I, Sequeira AF, et al. Recurrent duplications of the annexin A1 gene (ANXA1) in autism spectrum disorders. Mol Autism. 2014;5(1):28.

28 Pan X, Peng L, Yin G. Downregulation of Annexin A1 by short hairpin RNA inhibits the osteogenic differentiation of rat bone marrow-derived mesenchymal stem cells. Int J Mol Med. 2015;36(2): 406-14. 
29 Petrella A, Festa M, Ercolino SF, Zerilli M, Stassi G, Solito E, et al. Annexin-1 downregulation in thyroid cancer correlates to the degree of tumor differentiation. Cancer Biol Ther. 2006;5(6):643-7.

30 Ying X, Chen L, Xie J, Hu Y, Wu Q, Cao L, et al. ANXA1 (Annexin A1) regulated by MYC (MYC protooncogene) promotes the growth of papillary thyroid carcinoma. Bioengineered, 2021;12(2):9251-65.

31 Zhao X, Ma W, Li X, Li H, Li J, Li H, et al. ANXA1 enhances tumor proliferation and migration by regulating epithelial-mesenchymal transition and IL-6/JAK2/STAT3 pathway in papillary thyroid carcinoma. J Cancer. 2021;12(5):1295-1306.

32 Arora C, Kaur D, Naorem LD, Raghava GPS. Prognostic biomarkers for predicting papillary thyroid carcinoma patients at high risk using nine genes of apoptotic pathway. PLoS One. 2021;16(11):e0259534.

33 Feng J, Lu SS, Xiao T, Huang W, Yi H, Zhu W, et al. ANXA1 Binds and Stabilizes EphA2 to Promote Nasopharyngeal Carcinoma Growth and Metastasis. Cancer Res. 2020;80(20):4386-98.

34 Boudhraa Z, Merle C, Mazzocut D, Chezal JM, Chambon C, Miot-Noirault E, et al. Characterization of pro-invasive mechanisms and N-terminal cleavage of ANXA1 in melanoma. Arch Dermatol Res. 2014;306(10):903-14.

\section{Tables}

Table 1 Basic characteristics of the study samples. 


\begin{tabular}{|c|c|c|c|}
\hline $\begin{array}{l}\text { Demographic } \\
\text { characteristic }\end{array}$ & $\begin{array}{l}\text { N1b group } \\
n=6\end{array}$ & $\begin{array}{l}\text { N0 group } \\
n=6\end{array}$ & $P$ value \\
\hline Age(years) & $43.17 \pm 11.37$ & $49.67 \pm 10.03$ & 0.319 \\
\hline Gender & & & 1.00 \\
\hline Male & 3 & 3 & \\
\hline Female & 3 & 3 & \\
\hline Tumor size $(\mathrm{cm})$ & & & 1.00 \\
\hline$\geq 1$ & 4 & 3 & \\
\hline$\nabla 1$ & 2 & 3 & \\
\hline Multifocality & & & 1.00 \\
\hline Yes & 2 & 3 & \\
\hline No & 4 & 3 & \\
\hline TBSRTC & & & 1.00 \\
\hline II & 1 & 0 & \\
\hline III & 1 & 2 & \\
\hline IV & 0 & 0 & \\
\hline V & 1 & 2 & \\
\hline $\mathrm{VI}$ & 3 & 2 & \\
\hline
\end{tabular}

Figures 


\section{Papillary thyroid carcinoma tissues with N0 $(n=6)$ vs N1b $(n=6)$ Label-free quantitative proteomics}

\section{Identify differentially expressed proteins (DEPS) in PTC N0 vs PTC N1b}

\section{Bioinformatics analysis}

\section{Validated in the HPA database}

\section{Survival prognostic analysis in the GEPIA database}

Figure 1

Experimental design and workflow in this study. A label-free proteomic analysis was performed for PTC with or without lateral LNM tissues. The hub genes were identified and survival analysis was conducted for PTC with LNM. 


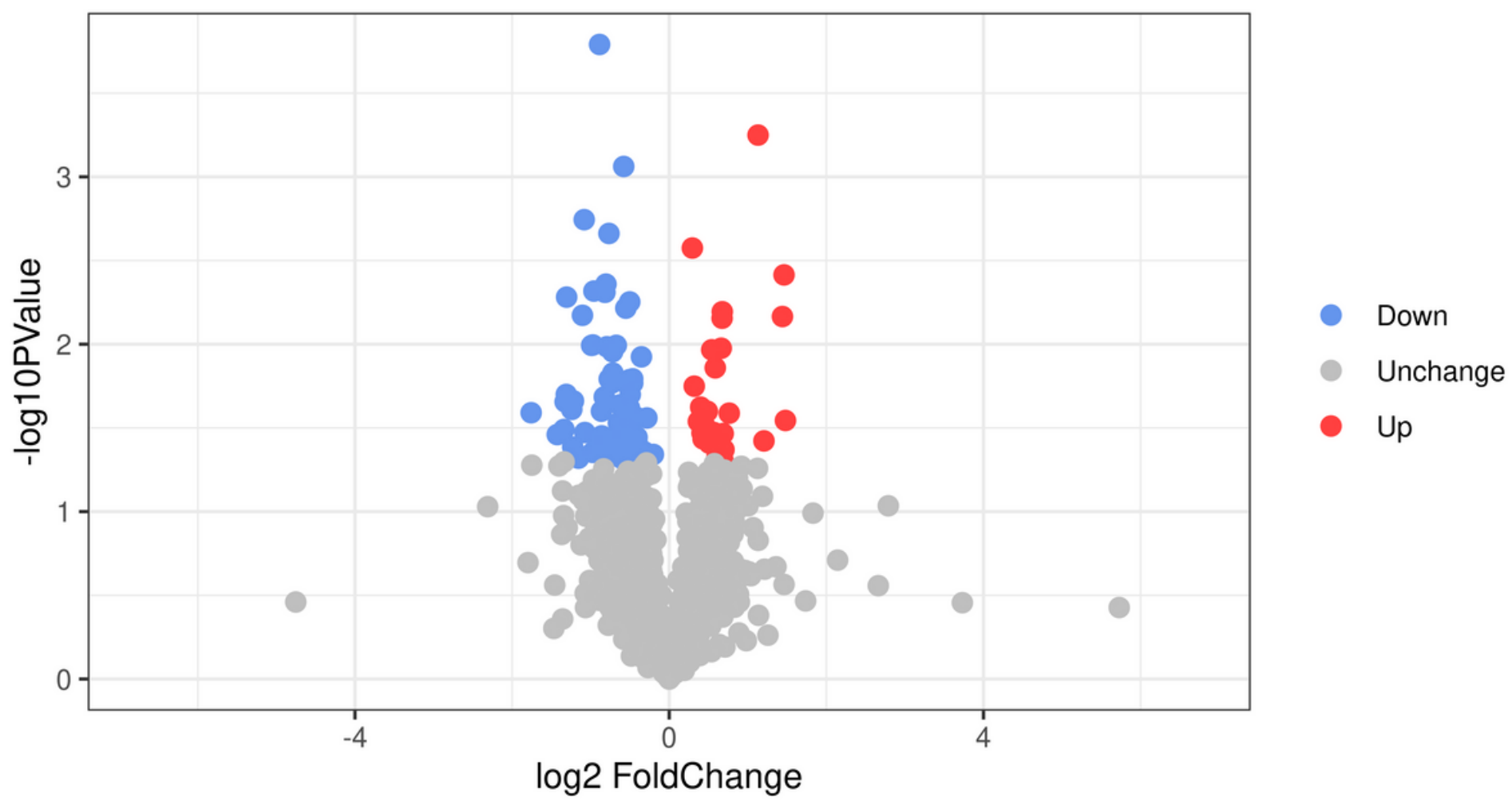

Figure 2

Proteins identified through proteomics. Based on the quantitative analysis, significant differential abundant proteins were illustrated by volcano plot. The -log10 (P value) is plotted against the log2 (fold N0 /N1b). 


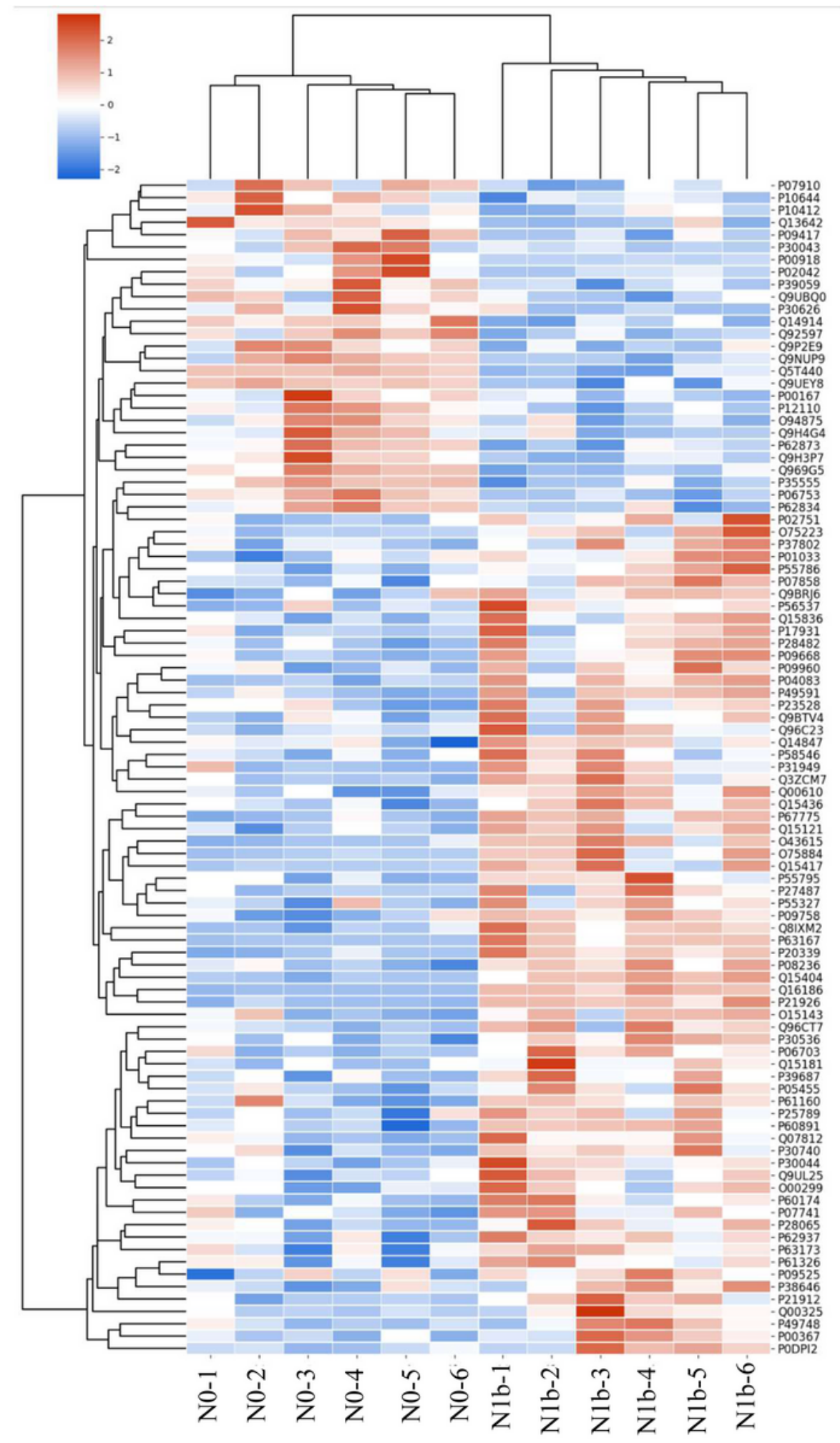

Figure 3

Hierarchical cluster analysis of 95 DEPs in PTC N0 groups and N1b groups. HCA of the twelve thyroid cancer samples (taken from twelve different patients) was shown in a heat map. HCA is shown on the left. 


\section{Figure 4}

Gene Ontology (GO) analysis on proteins of PTC N0 group and N1b group. GO functional included biological processes (BP), cell components (CC), as well as molecular function (MF).

\section{Figure 5}

KEGG pathway analysis indicated the top 10 signaling pathways enriched by the DEPs $(P<0.05)$. The dot size stands for the number of differentially proteins enriched in the corresponding pathway.

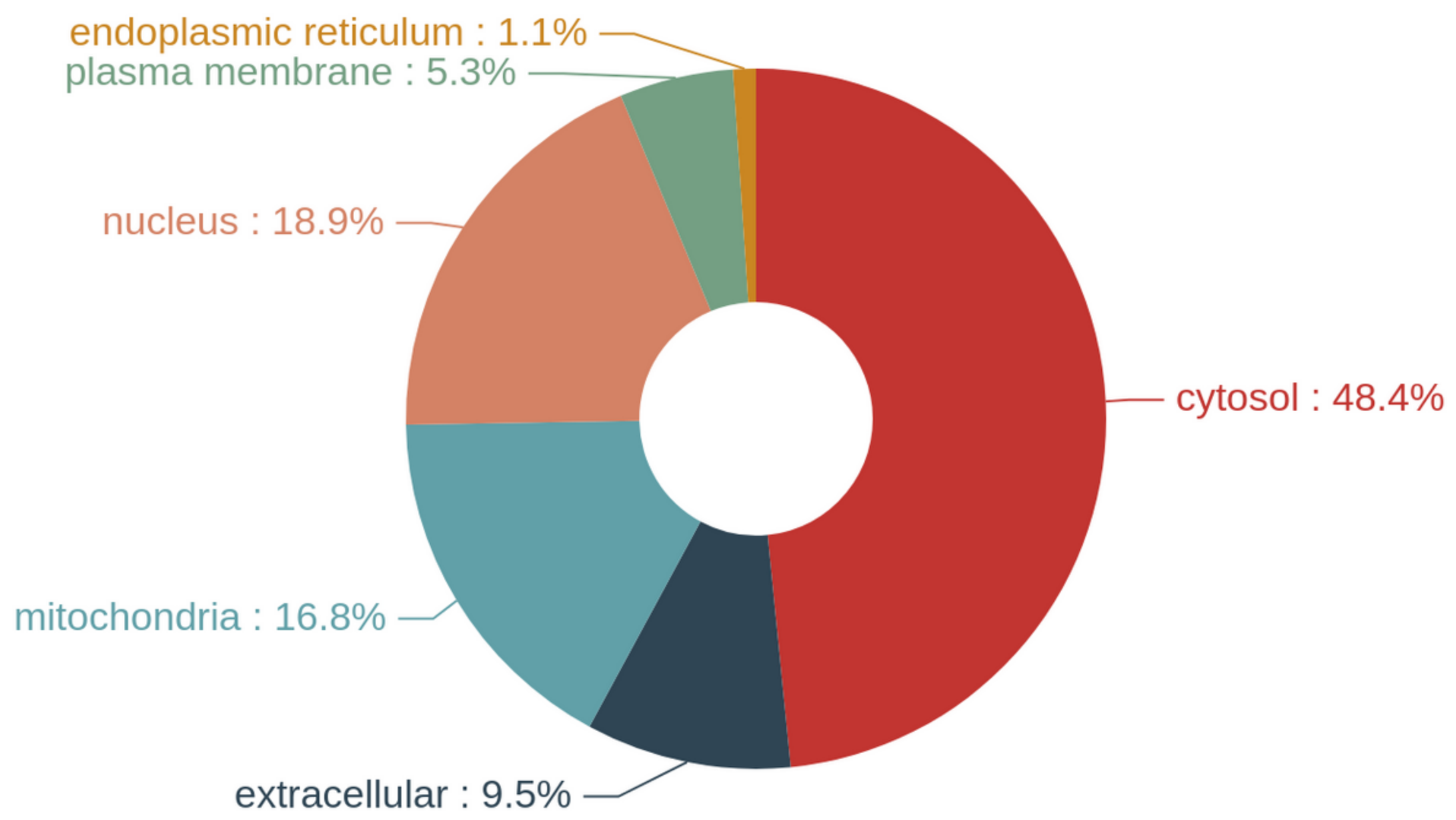

Figure 6

Subcellular localization of the DEPs. Different colors stand for different localities.

\section{Figure 7}

PPI network in PTC. Circles: genes are; lines: interactions between gene-encoded proteins; line colors: the evidence of interactions between proteins. 


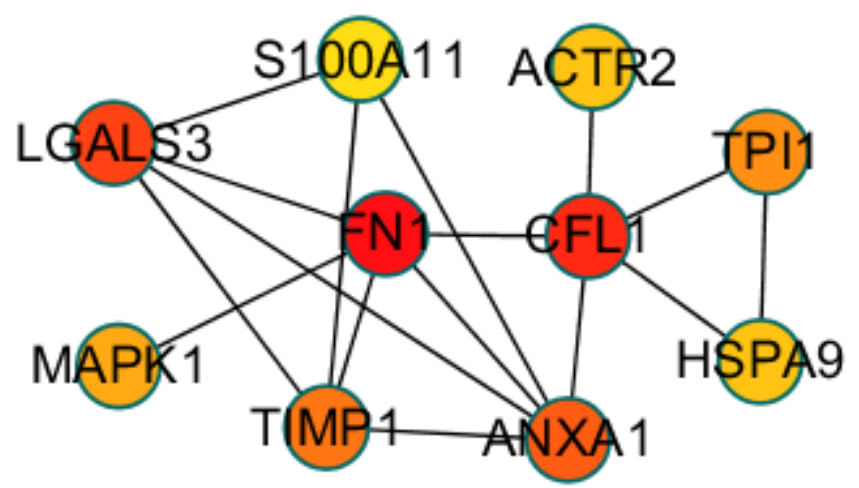

Figure 8

Interconnection of 10 hub genes; deeper color stands for a higher degree score. 


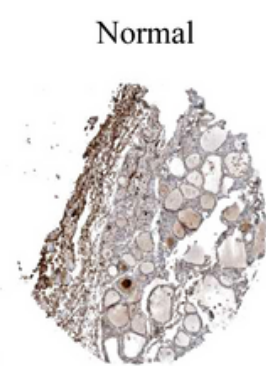

FN1 patient ID:3005 HPA027066 staining: Low

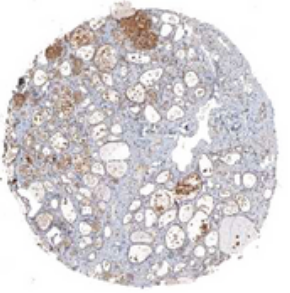

LGALS3 patient ID:1501 HPA003162 staining: Medium

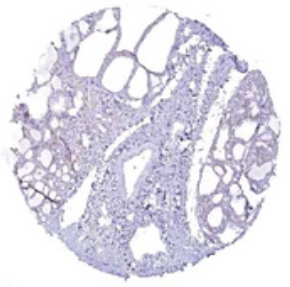

TIMP1 patient ID:1712 HPA053417 staining: Not detected
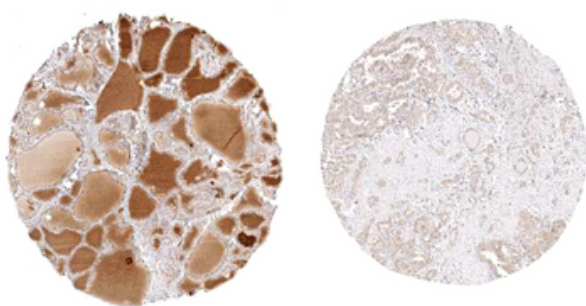

MAPK1 patient ID:3267

MAPK1 patient ID:1712 HPA030069 staining: Low HPA030069 staining: Medium
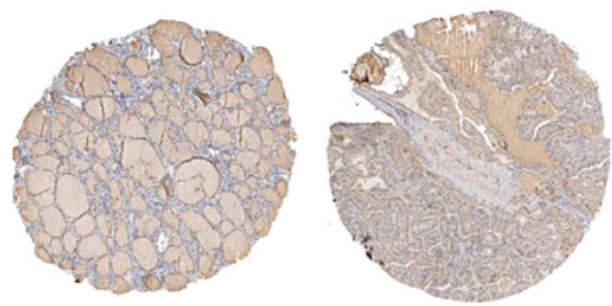

ACTR2 patient ID:2623 CAB005083 staining: Medium CAB005083 staining: Medium HPA042745 staining: Not detected

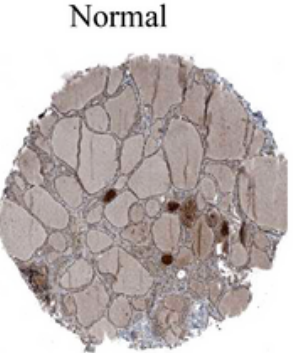

CFL1 patient ID:1712 CAB037077 staining: Low

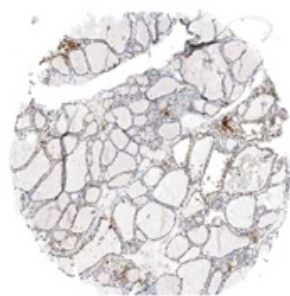

ANXAl patient ID:1712 CAB035987 staining: Low
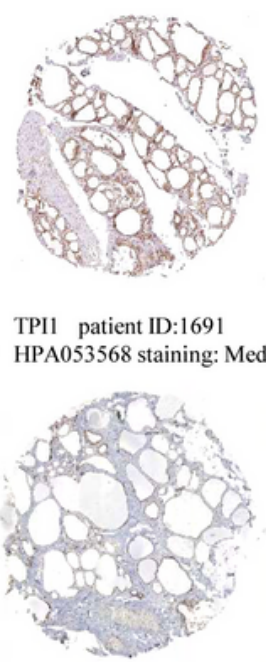

HSPA9 patient ID:1436 HPA000898 staining: High

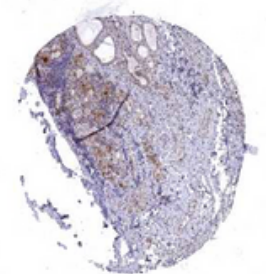

S100A11 patient ID: 1948

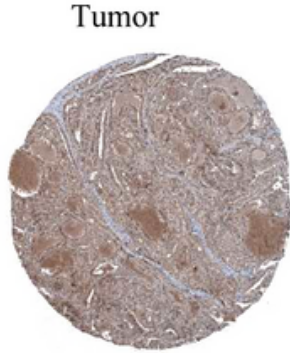

CFL1 patient ID:2623 CAB037077 staining: Medium

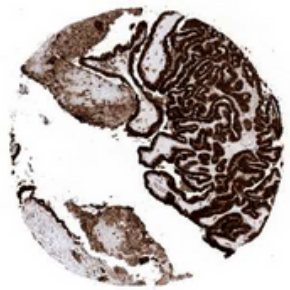

ANXA1 patient ID:2623 CAB035987 staining: High

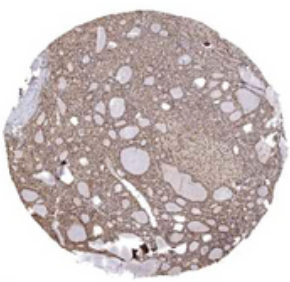

TPIl patient ID:4236 HPA053568 staining: Medium

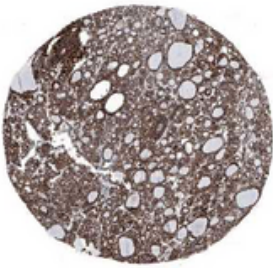

HSPA9 patient ID:923 HPA000898 staining: High

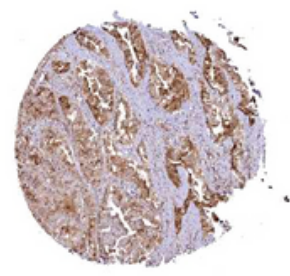

S100A11 patient ID:3037 HPA042745 staining: High

\section{Figure 9}

The histological expressions of hub genes. The category of tissue specimen was noted on the top of the figure. The gene's name, antibody type, patient ID, as well as staining degree are all listed at the bottom of each image. 
Figure 10

Kaplan-Meier analysis for hub genes. Two hub genes were correlated with the prognosis of PTC $(P<0.05)$. (a) The Disease-Free Survival (DFS) of FN1 in GEPIA database. (b) The Overall Survival (OS) of ANXA1 in GEPIA database. 